\title{
Relationship of trait anger with quality of life and anger control among alcohol users
}

\author{
Manoj Kumar Sharma ${ }^{1 *}$ L. N. Suman ${ }^{1}$, Pratima Murthy ${ }^{2}$, P. Marimuthu ${ }^{3}$ \\ ${ }^{1}$ Department of Clinical Psychology, NIMHANS, Bangalore, India \\ ${ }^{2}$ Centre for Addiction Medicine, Department of Psychiatry, NIMHANS, Bangalore, India \\ ${ }^{3}$ Department of Biostatistics, NIMHANS, Bangalore, India \\ Email: mks712000@yahoo.co.in
}

Received 14 August 2012; revised 16 September 2012; accepted 27 September 2012

\begin{abstract}
Background: Abstinence and anger control are promoted as ways to improve quality of life. Trait anger has been seen as one casual factor for alcohol related aggression and poor quality of life. The present study assesses anger control, quality of life and its relation with trait anger among alcohol dependents, abstainers \& social drinkers. Material \& Method: 150 subjects (50 subjects in each group) in the age range of 20 45 years were taken for the study. Semi-structured interview schedule, State-Trait Anger Expression Inventory-2, WHOQOL-BREF were administered in individual setting. Results: Dependent alcohol users and abstainers reported the significant impact of alcohol use on family \& occupational functioning. Quality of life has correlation with anger control (in/out), trait anger, temperament $\&$ reaction in the control $\&$ abstainers group, whereas quality of life has negative correlation with trait anger expression among the alcohol group. Conclusions: Alcohol use has been associated with the trait anger expression and poor quality of life. It has implications for promoting alcohol abstinence, in improving quality of life and in anger control.
\end{abstract}

Keywords: Trait Anger; Anger Control; Quality of Life

\section{INTRODUCTION}

Anger is a social emotion, its expression invariably has a consequences to social relations within a culture or society. Culture based expectations also influence the expression and control of anger. Indian participants use more introspective strategies like repression and rational self coping statements to manage anger provocation than their western counterparts [1]. Gender and location of participants also has a significant correlation with the anger expres-

\footnotetext{
${ }^{*}$ Corresponding author.
}

sion index [2]. In a study comparing anger expression and control carried out on Iranian and Indian patients (200 in each group) using state trait anger expression inventory \& daily hassel checklist. Iranian male students scored higher on anger expression index whereas Indian males scored higher on anger control [3]. Drug users experience anger more frequently than nonusers and they are more likely to feel less in control of their angry feelings. They are more apt to express such anger toward other persons or objects in their environment [4]. Alcohol interferes with those processes which are needed to control behaviour and act in a planful way to achieve the best outcomes in any situation, namely attention, abstracting relevant information, reasoning, problem-solving, planning, and self regulation [5] and one specific set of skills to which these processes are integral is social problem solving [6].

Participants with high trait anger are more prone to use alcohol and drugs [7]. Trait anger has an association with aggression among intoxicated men who reported low level of anger control [8]. Alcohol use was reported more among people with moderate levels of trait anger [9]. Abstinence, controlled or minimal drinking has positive impact on quality of life among alcohol users [10].

In a culture like India where abstinence is generally the desired goal, there is a need to understand the influence of alcohol on trait anger, anger control and quality of life. The present work assesses anger control, quality of life and its relation with trait anger among alcohol dependents, life time abstainers and social drinkers.

\section{METHODS}

\subsection{Participants \& Procedure}

The study was reviewed and approved by the NIMHANS Ethics Committee. The total study sample comprised 150 subjects in the age range of 20 - 45 years. Group I consisted of 50 patients with active alcohol dependence drawn from both outpatient and inpatient settings. Group II consisted of 50 who had been diagnosed of 
alcohol dependence but currently maintaining abstinence from alcohol for the last three months. Group III comprised of 50 social drinkers (no lifetime use of more than 2 standard drinks per day). This group was drawn from the community (20 - 45 years of age and gender matched samples drawn from among the hospital staff, educational institutions and workplaces using a snowballing approach). Subjects who had organic disorders, poly substance use (except nicotine), psychosis or sensory deficits were excluded from the study. Each assessment session lasted about 30 minutes and all the subjects were assured of confidentiality.

\subsection{Measurement}

A Semi-structured interview schedule was developed by the investigators to obtain information about socio-demographic details, information about alcohol use, its relationship with anger and its effects on anger control. StateTrait Anger Expression Inventory-2 [11], WHOQOLBREF [12] were used.

\subsection{Statistical Analysis}

It was carried out using percentage of score and factor analysis.

\section{RESULTS}

While $76 \%$ of social users and $68 \%$ of active alcohol dependent users perceived anger as a negative emotion, these differences were not statistically significant. A majority of the dependent users (52\%), abstainers (46\%) attributed anger to personal reasons (persisting irritation, frustration, negative attitude toward the alcohol users, decrease communication with others). $60 \%$ of the dependent users and abstainers experienced it as having a significant impact on the family (decrease communication with wife, frequent fights) and workplace area (loss of job and conflict with employers).

Factor analysis was carried out to understand the correlated factor in each of the three groups (Table 1). An eigen value greater than 1 was selected for the factor to be considered and the correlation greater than 0.33 was considered for the rotated component matrices and the rotation was done using varimax method. In Table 1, Columns 1 to 3 is rotated matrix from the factor analysis for the control group. Three factors (trait anger, trait anger reaction and trait anger temperament) are seen to have a high correlation with total quality of life scores, the total quality of life alone explains $42.88 \%$ of the variability of correlation and the trait anger correlation (with anger control in and anger control out) explains $27.02 \%$ and therefore, a total of $69.9 \%$ of the variability is explained by the total quality of life scores and trait anger in the control group.

Columns 4 to 6 in Table 1 are the rotated matrix from the factor analysis for the Abstainers group. Here two factors (trait anger, trait anger reaction) have a high correlation with total quality of life score, and this explains the $47.24 \%$ of the variability in the correlation. Trait anger is also highly correlated with the three factors (total quality of score, anger control out, anger control in) which explains $24.54 \%$ of the variability. Therefore two factors, viz. the total quality of life score and the trait anger score both explained most of the variability (71.78\%) in the correlation matrix.

For the alcohol dependent group (Columns 6 and 7), it is only the trait anger reaction score which explain the correlation with the three variables (that is anger control out and anger control in and trait anger expression with a negative correlation). It is the trait anger reaction score which alone explain almost $50 \%$ of variability in the correlation matrix. If we consider the anger control out also as a factor. then the total variability explained would be $73.8 \%$. Since the eigenvalue of the anger control out is $(0.955)$ less than 1 , this factor is not selected for the further analysis.

\section{DISCUSSION}

In this study, we found that the quality of life has a positive correlation with anger control (in/out), trait anger, temperament and reaction in the control and abstainers group, whereas quality of life has a negative correlation

Table 1. Factor analysis for three group controls, abstainers and alcohol (rotated matrix) for quality of life (QUL), trait anger, trait anger reaction, anger control out, anger control in \& trait anger temperament.

\begin{tabular}{|c|c|c|c|c|c|c|c|}
\hline \multirow{2}{*}{$\frac{\text { Controls }}{(1)}$} & \multirow[b]{2}{*}{ Total QOL (2) } & \multirow[b]{2}{*}{ Trait anger (3) } & \multirow{2}{*}{$\begin{array}{l}\text { Abstainers } \\
\text { Total QOL (4) }\end{array}$} & \multirow[b]{2}{*}{ (5) } & \multirow{2}{*}{\multicolumn{2}{|c|}{$\begin{array}{ll} & \text { Alcohol } \\
\text { Trait anger (6) (7) }\end{array}$}} & \multirow[b]{2}{*}{ Total QOL (8) } \\
\hline & & & & & & & \\
\hline Total QOL & & & Tot QOL & & 0.412 & Tot QOL & \\
\hline Trait anger & 0.962 & & Trait anger & 0.918 & & Trait anger & \\
\hline Trait anger reaction & 0.864 & & Trait anger reaction & 0.963 & & Trait anger reaction & -0.584 \\
\hline Anger control out & & 0.895 & Anger control out & & 0.866 & Anger control out & 0.845 \\
\hline Anger control in & & 0.876 & Anger control in & & 0.866 & Anger control in & 0.827 \\
\hline Trait anger temperament & 0.882 & & Trait anger Temperament & & & Trait anger expression & -0.507 \\
\hline
\end{tabular}


with trait anger expression among the active alcohol dependent users. Dependent users and abstainers reported a significant impact of alcohol use on family and occupational functioning. This indicates that alcohol use influences both quality of life and anger control (in/out). Anger was perceived as negative emotion across the three groups.

Our findings have been corroborated by other studies as well. Interactive effect of trait anger and alcohol consumption on anger control was seen in the intoxicated state. Whereas same relationship was not found between alcohol consumption and trait anger in the self reported sober state [13]. Alcohol also exerts proximal effects on abusive behavior among individuals already prone to respond to conflict with increased anger [14]. Males who abuse alcohol experience and express more anger than males who do not abuse alcohol [15]. Alcohol users experience lower quality of life in comparison to non alcohol users [16]. Abstinence or moderate consumption also leads to improved quality of life [17]. It is also associated with long term recovery [18]. Longitudinal research has shown that early impulsivity is associated with both later offending \& problem drinking and it is also related with poor executive functioning which in turn contributes to aggressiveness [19]. Thus, in a culture which promotes abstinence and anger restraint, alcohol use enhances the expression of trait anger. These finding have implications for psychoeducating people for abstinence from alcohol, its role in improving the quality of life as well as for anger control. Future research in this area should focus on male and female samples, trait anger expression and its relationship with alcohol use, resilience to stress, domestic violence, relation with relapse and psychological comorbidity.

\section{ACKNOWLEDGEMENTS}

Grant agency: Research support from the Center for Addiction Medicine, NIMHANS, Hosur Road, Bangalore, Karnataka, India, awarded to Dr. Manoj Sharma.

Mrs Sowmya, HR, Research Assistant, for data collection \& writing the report.

\section{REFERENCES}

[1] Suchday, S. and Larkin, K.T. (2004) Psychophysiological responses to anger provocation among Asian, Indian, and white men. International Journal of Behavioral Medicine, 11, 71-80. doi:10.1207/s15327558ijbm1102 2

[2] Neele, K. (2011) A cross-cultural study of anger in relation to family, gender and stress. http://independent.academia.edu/DrNeelkonwar/Papers/3 $\underline{56034 / 2010}$

[3] Moheb, N. and Ram, U. (2010) Cross cultural study of stress and anger. Procedia Social and Behavio, 5, 1765-
1769. doi:10.1016/j.sbspro.2010.07.361

[4] De Moja, C.A. and Spielberger, R.C.D. (1997) Anger and drug addiction. Psychological Reports, 81,152-159. doi:10.2466/pr0.1997.81.1.152

[5] Pihl, R.O. and Hoaken, P.N.S. (2002) Biological bases of addiction and aggression in close relationship. In: Wekerle, C. and Wall, A.M., Eds., The Violence and Addiction Equation, Brunneer-Routledge, New York, 25-43.

[6] Zelazo, P.D., Carter, A., Resnck, J.S. and Frye, D. (1997) Early development of executive function: A problem solving frame work. Review of General Psychology, 1, 198-226. doi:10.1037/1089-2680.1.2.198

[7] Tafrate, R.C., Kassinove, H. and Dundin, L. (2002) Anger episodes in high and low trait anger community adults. Journal of Clinical Psychology, 58, 1573-1590. doi:10.1002/jclp.10076

[8] Parrott, D.J. and Giancola, P.R. (2004) A further examination of the relation between trait anger and alcohol-related aggression: The role of anger control. Alcohol: Clinical and Experimental Research, 28, 855-864. doi:10.1097/01.ALC.0000128226.92708.21

[9] Parrott, D.J. and Zeichner, A. (2002) Effects of alcohol and trait anger on physical aggression in men. Journal of Studies on Alcohol and Drugs, 63, 196-204.

[10] Foster, J.H., Powell, J.E., Marshall, E.J. and Peter, T.J. (1999) Quality of life in alcohol-dependent subjects-A review. Quality of Life Research, 8, 255-261. doi:10.1023/A:1008802711478

[11] Spielberger, D.C. (1999) STAXI-2 state trait anger expression inventory-2, professional manual. PAR, Florida.

[12] World Health Organization (1996) WHOQOLF-BREF: Introduction, administration, scoring and generic version of assessment. WHO, Geneva.

[13] Johansson, A., Santtila, P., Corander, J., Jern, P., Von der Pahlen, B., Varionen, M. and Sandabba, K. (2011) Controlling anger in self reported sober and alcohol intoxicated states: Moderting effects of trait anger and alcohol consumption. Scandinavian Journal of Psychology, 52, 382-388. doi:10.1111/j.1467-9450.2011.00880.x

[14] Eckhardt, C.I. (2007) Effects of alcohol intoxication on anger experience and expression among partner assaultive. Journal of Consulting and Clinical Psychology, 75, 61-71. doi:10.1037/0022-006X.75.1.61

[15] Willard, S. (1999) Anger and alcohol use: A model of coping styles, alcohol expectancies and the experience and expression of anger. Masters Theses \& Specialist Projects, 763. http://digitalcommons.wku.edu/theses/763

[16] Welsh, J.A., Buchsaum, D.G. and Kaplan, C.B. (1993) Quality of life of alcoholic and non alcoholic: Does excessive drinking make a difference in the urban setting. Quality of Life Research, 2, 335-340. doi:10.1007/BF00449428

[17] Gillet, C., Pailleam, F., Wahla, D., Aubinb, H.-J., Pirolleta, P. and Prime, T. (1991) Outcome of treatment in alcoholic women. Drug Alcohol Dependence, 29, 189-194. doi:10.1016/0376-8716(91)90048-4

[18] Chaturvedi, S., Kirthana, R. and Desai, M. (1997) Quality 
of life and alcoholism: What aspects are perceived as important. Quality of Life Research, 6, 632.

[19] Kliteberg, B.A., Anderson, T., Magnusson, D. and Stattin, H. (1993) Hyperactive behavior inn childhood as related to subsequent alcohol problems and violent offending: A longitudinal study of male subjects. Personality and Individual Differences, 15, 381-388. 